\title{
Identification of a Novel Nonsense Homozygous Mutation of LINS1 Gene in Two Sisters With Moderate Intellectual Disability, Schizophrenia, and Anxiety Disorders and Review of the Literature
}

Chia-Hsiang Chen ( $\nabla$ cchen3801@gmail.com )

Department of Psychiatry, Chang Gung Memorial Hospital, Linkou, Taoyuan, Taiwan 2Department and 3 Graduate Institute of Biomedical Sciences, Chang Gung University, Taoyuan, Taiwan

https://orcid.org/0000-0002-8232-2403

\section{Yu-Shu Huang}

Chang Gung Memorial Hospital Linkou Main Branch: Chang Gung Memorial Hospital

Ting-Hsuan Fang

Chang Gung University

\section{Research}

Keywords: LINS1, Intellectual disability, schizophrenia, anxiety, whole genome sequencing

Posted Date: September 16th, 2020

DOI: https://doi.org/10.21203/rs.3.rs-76249/v1

License: (1) This work is licensed under a Creative Commons Attribution 4.0 International License.

Read Full License 


\section{Abstract}

Background: The human LINS1 gene is located at 15q26.3 and encodes the lines homolog 1 protein that contains the Drosophila lines homologous domain. Mutations in the LINS1 gene have been reported to cause a rare recessive form of intellectual disability. A total of seven mutations in six studies have been reported in the literature to our knowledge.

Results: Using whole genome sequencing analysis, we identified a novel homozygous nonsense mutation in the LINS1 gene in these two sisters who presented moderate intellectual disability, schizophrenia symptoms, and severe anxiety. The mutation was a C-to-T substitution at the CDNA nucleotide position 274 that changed the amino acid glutamine at the codon 92 to stop codon (Gln92X), resulting in the truncation of LINS1 protein after amino acid 91. This mutation was transmitted from their unrelated parents who were heterozygous carriers.

Conclusions: Our findings not only add to the allelic heterogeneity of the LINS1-associated intellectual disability but also expand the spectrum of clinical phenotypes of patients with LINS1 mutations. Our study suggests that the human LINS1 gene mutation may play a role in the pathogenesis of psychosis and anxiety in addition to the intellectual disability.

\section{Background}

Intellectual disability is a childhood-onset developmental disorder defined by the reduced intelligent function that leads to impaired learning ability and social adaptation. The prevalence of intellectual disability is approximately $1 \%$ in the general population worldwide (1). Intellectual disability is a complex disorder with the involvement of genetic and environmental factors, but the genetic factor is the leading cause of intellectual disability (2). The genetic basis of intellectual disability is very heterogeneous, ranging from chromosomal abnormalities, copy number variations to single-gene mutations $(3,4)$. And the inheritance mode of genetic mutation may include de novo mutation, Mendelian inheritance, and nonMendelian inheritance in different patients. Intellectual disability is further divided into syndromic and non-syndromic intellectual disability, depending on whether there is the co-existence of distinguishable physical abnormalities (2). Several hundreds of genes related to intellectual disability have been identified, however, many more remain to be identified $(2,5)$. Hence, it is a challenging task to establish the correct genetic diagnosis for the affected patients in clinical settings to offer genetic counseling and to guide the clinical treatment (6). Recently, the advancement of next generation sequencing technology has greatly facilitated the progress in this aspect $(7,8)$.

The LINS1 gene is located at 15q26.3 and encodes the lines homolog 1 protein that contains the Drosophila lines homologous domain (9). Drosophila lines protein encoded by the segment polarity gene lin functions as a tissue- and stage-specific modulator of Wnt/Wingless-dependent patterning of the dorsal epidermis in Drosophila (10). Rare mutations of the LINS1 gene have been linked to a recessive type of intellectual disability. Najmabadi and colleagues studied 136 consanguineous families with 
autosomal-recessive intellectual disability and found a homozygous deletion of four nucleotides in exon 5 of the LINS1 gene in one consanguineous family (11). Although they originally thought the gene was without an obvious link to intellectual disability, later studies showed more evidence to support the link between the LINS1 gene mutations and recessive form of intellectual disability. Akawi and colleagues reported a homozygous splicing mutation of the LINS1 gene in a consanguineous family with two children affected with intellectual disability (12). Sheth and colleagues also discovered a homozygous missense mutation E313K of the LINS1 gene in two siblings presenting non-syndromic intellectual disability and mutism (13). Recently, Muthusamy and colleagues identified a homozygous nonsense mutation (Q368X) of the LINS1 gene in two siblings with non-syndromic intellectual disability in a consanguineous family (14). Chang and colleagues also reported a homozygous single nucleotide deletion of the LINS1 gene that led to frameshift mutation (D241fs) of the LINS1 gene in a child with severe intellectual disability (15). Together, these data suggest that loss-of-function mutations of the LINS1 gene are rare, but link to the recessive form of non-syndromic intellectual disability.

In our molecular genetic study series of neuropsychiatric disorders, we studied a family with two affected sisters who were diagnosed with moderate intellectual disability since childhood. These two sisters also manifested psychiatric symptoms that met the diagnosis of schizophrenia and anxiety disorders in their thirties. We conducted a whole-genome sequencing analysis for this family and detected a novel nonsense homozygous mutation of the LINS1 gene in these two affected sisters. Here, we report the clinical characteristics and molecular genetic findings of this family.

\section{Materials And Methods}

\section{Subjects}

The Taiwanese family was recruited from the outpatient clinic of the Department of Psychiatry, ChangGung Memorial Hospital-Linkou, Taoyuan, Taiwan. The study was approved by the Institutional Review Board of Chang-Gung Memorial Hospital with the approved number of 201801385A3. Clinical data of the patients were collected based on interviews with the family and reviews of their medical records. Informed consent was obtained from each subject after a full explanation of the study. Genomic DNA was prepared from each subject using the method established in the laboratory.

\section{Wholegenome sequencing}

Paired-end whole-genome sequencing was performed using the Illumina NovaSeq6000 platform (Illumina, San Diego, CA, USA) according to the standard protocol provided by the company. After a quality check, the raw data were aligned to the human reference genome build hg19. SAMtools and Genome Analysis Tool Kit were used to refine the local alignment and to generate variant calling file (VCF) for each subject. Variants were further annotated, filtered, and analyzed under different inheritance models, including autosomal dominant, autosomal recessive, $X$-linked, and de novo mutation. The bioinformatics and family analyses were implemented using SeqsLab software (Atgenomix, Taipei, Taiwan). 


\section{Sanger sequencing}

To verify the authenticity of the LINS1 mutation, we designed a pair of primers (LINS1-F:5'-GCA CTG TGC AGC CAT GTG AGA-3' and LINS1-R:5'-GGG CAA ACA CCT GTG GTA TCC-3') to obtain an amplicon of 399 bp that covered the mutation by polymerase chain reaction (PCR). In brief, 35 cycles of PCR were performed in a $20 \mathrm{ml}$ mixture containing $100 \mathrm{ng}$ DNA, $1 \mathrm{mM}$ of each primer, $1 \mathrm{X}$ buffer, I $\mathrm{ml}$ of $2.5 \mathrm{mM}$ of dNTP, $0.5 \mathrm{U}$ of Power Taq polymerase. An aliquot of the amplicon was purified and subjected to Sanger sequencing using the BigDye Terminator kit v3.1 (Applied Biosystems, Foster, CA, USA).

\section{Bioinformatics analysis}

The frequency of the mutation identified in this study was checked in the dbSNP (https://www.ncbi.nlm.nih.gov/snp/) and the Taiwan Biobank (https://taiwanview.twbiobank.org.tw/index). The prediction of the functional impact of the nonsense mutation identified in this study was assessed using the web-based MutationTaster2 (http://mutationtaster.org/).

\section{Results}

\section{Clinical report}

The pedigree of this family is shown in Figure 1A. The sisters (CG1214, CG1215) were in their forties (CG1215) when they first visited our psychiatric outpatient unit. They were accompanied by their parents (CG1216 and CG1217) who were in their sixties. According to their unrelated parents, both sisters were delivered smoothly at full term with uneventful pregnancies. However, they were found to have a delay in developmental milestones, especially speech development, hence, they were diagnosed with moderate intellectual disability with unknown etiology. They did not have other physical abnormalities nor seizure history. They received special education from elementary to middle school. Nevertheless, they did not have enough social function to support themselves. They did not have a job after graduation from school and stayed with their parents. Both sisters started to manifest psychotic symptoms such as auditory hallucination, self-talking, a delusion of persecution, bizarre behavior, and wandering around aimlessly in their thirties. Also, they expressed severe anxiety in addition to their psychotic features. Hence, they were diagnosed with moderate intellectual disability comorbid with schizophrenia and anxiety disorders. They were treated with both antipsychotics and anxiolytics with a fair response. Their psychotic symptoms and anxiety improved and remained stable under long-term medication.

\section{Whole-genome sequencing and Sanger sequencing}

Under the recessive model of analysis of their whole genome sequence data, we identified a G-to-A homozygous mutation at the genomic DNA position 101120774 (hg19) of the LINS1 gene in these two sisters. This mutation led to a C-to-T substitution at the cDNA position 274 , which resulted in an amino acid change from glutamine to stop codon at the protein sequence 92, designated GIn92X. Both parents 
were heterozygous carriers of this mutation. The authenticity of this mutation in this family was verified using Sanger sequencing. The chromatograms of the mutation in this family are shown in Figure 1B-1E.

\section{Bioinformatics analysis}

This G-to-A mutation was already present in the dbSNP and was assigned rs755894515. The allele frequency of this mutation in the current release (20200227123210) of Allele Frequency Aggregator (ALFA) is 0, 0.000080 in Genome Aggregation Database (GenomAD_exome), and 0.000074 in Exome Aggregation Consortium (ExAC). The allele frequency of this allele in Taiwan Biobank is 0.002 . The webbased software MutationTaster2 predicted that the nonsense mutation was disease-causing.

\section{Discussion}

In this study, we identified a novel homozygous mutation of the LINS1 gene (Gln92X) in two sisters diagnosed with moderate intellectual disability since childhood. They did not have seizure history or other distinguishable physical abnormalities or facial dysmorphisms, hence, their intellectual disability diagnosis can be classified as non-syndromic. To our knowledge, six studies have reported the developmental conditions associated with LINS1 mutations in the literature (11-16). All the patients had a global developmental problem and intellectual disability in common and most of them were diagnosed with non-syndromic intellectual disability, given that there were some variations in clinical phenotype in these patients. McMillan and colleagues reported a male patient manifesting longstanding drooling, dysphagia, and impaired tongue movement that met the diagnosis of Worst-Drought syndrome, a congenital pseudobulbar paresis in addition to intellectual disability (16). So far, a total of 8 mutations of the LINS1 gene were identified from these studies including ours. All these mutations are rare mutations and private for each affected family, suggesting the high allelic heterogeneity of LINS1 mutations associated with intellectual disability. We summarize the location of these mutations in Figure 2.

Notably, our patients developed schizophrenia and anxiety disorders in their thirties. They did not have behavioral problems that need psychiatric intervention before the onset of their psychiatric conditions. Approximately one-third of the people diagnosed with intellectual disability were reported to have cooccurrence of psychiatric disorders $(17,18)$, with the over-representation of the diagnosis of schizophrenia (18). In the case of LINS1-associated intellectual disability, Najmabadi and colleagues did not report any behavioral phenotypes in their patients (11). Akawi and colleagues reported extreme hyperactivity and aggressive behavior that needed medication in one patient ( 9 years old) manifested, but not in his affected younger sister (3 years old) (12). Seth and colleagues observed autistic features in a male patient ( 21 years old), but they did not mention the autistic features in the affected elder brother (33 years old) (13). To our knowledge, we are the first to report the co-morbidity of schizophrenia, anxiety disorder, and intellectual disability in patients with LINS1 mutation. Our observation suggests that the LINS1 gene may play a role in the pathogenesis of psychiatric conditions in addition to its participation in the pathogenesis of intellectual disability. 
Human LINS1 protein comprises 757 amino acids and is expressed in various tissues including adult testis, prostate, spleen, thymus, skeletal muscle, fetal kidney, and brain (9). The LINS1 protein contains the Drosophila Lin homologous domain located from amino acid 378 to 538 . Our mutation (GIn92X) is a nonsense mutation, and presumably would lead to truncation of the LINS1 protein after amino acid 91, including the Drosophila Lin homologous domain. Hence, this mutation would be a loss-of-function mutation. In the bioinformatics analysis, this mutation was predicted to be a disease-causing mutation by the software Mutation Taster.

The physiological function of the LINS1 gene in human development is still unclear. Studies of Lines gene, the homolog of LINS1 gene in Drosophila, showed that the Lines gene was involved in the development of several tissues and organs such as wing (19), for- and hindgut (20), and epidermis (21). Hatini and colleagues demonstrated that the Lines gene was essential for tissue- and stage-specific Wnt signaling events in the cell-fate specification, activation of Wnt signaling promoted entry of the lin protein that was encoded by the Lines gene into nuclei (10). This finding suggests that the human LINS1 protein might be involved in the WNT signaling pathway as well.

WNT signaling is essential for embryonic development in all animals (22). There are several pathways in WNT signaling, including canonical and non-canonic pathways. Mutations of genes encoding the proteins involved in the WNT signaling have been linked to several human diseases, such as hereditary colorectal cancer and various types of sporadic cancers, bone disease, vascular diseases, etc (23). WNT signaling also plays an essential role in regulating neuronal connectivity in the nervous system $(24,25)$. Hence, disturbances of WNT signaling due to mutations of genes involved in the WNT signaling pathway have been associated with several neurodevelopmental disorders (23), such as congenital hydrocephalus (26), microcephaly (27), and intellectual disability $(28,29)$. Furthermore, WNT signaling has been reported to be associated with neuropsychiatric disorders including autism $(28,30)$, bipolar disorder $(31,32)$, schizophrenia $(33,34)$, and Alzheimer's disease $(35)$. In this context, we suggest that mutations of the LINS1 gene may interfere with the integral development of neurons in the brain and lead to intellectual disability and other developmental and neuropsychiatric conditions. Further studies are needed to elucidate the details of the pathogenesis.

\section{Conclusions}

We report the identification of a novel nonsense mutation of the LINS1 gene in two sisters with moderate intellectual disability co-morbid with schizophrenia and anxiety disorders. Our findings not only expand the clinical phenotypes associated with LINS1 mutations but also implicate the important role of the LINS1 gene in the development of cognition and mental function.

\section{List Of Abbreviations}

ALFA: Allele Frequency Aggregator, GenomAD: Genome Aggregation Database ExAC: Exome Aggregation Consortium 


\section{Declarations}

\section{Ethics approval and consent to participate}

The study was approved by the Institutional Review Board of Chang-Gung Memorial Hospital with the approved number of 201801385A3. Informed consent was obtained from each subject after a full explanation of the study.

\section{Consent for publication}

Not applicable.

\section{Availability of data and materials}

All data generated or analyzed during this study are included in this article

\section{Competing interests}

All the authors declare no conflict of interest.

\section{Funding}

The study was supported by grants CORPG3J0521 and CORPG3J031 from Chang Gung Memorial Hospital-Linkou, Taoyuan, Taiwan. The funding body did not involve in the design of the study and collection, analysis, and interpretation of data and in writing the manuscript.

\section{Authors' contributions}

$\mathrm{CHH}$ and YUH designed and supervised the study. TSF conducted the experiments and analyzed the data.

\section{Acknowledgements}

We thank the staff from ATGENOMIX (Taipei, Taiwan) for their assistance in the analysis of whole genome sequencing data.

\section{References}

1. Maulik PK, Mascarenhas MN, Mathers CD, Dua T, Saxena S. Prevalence of intellectual disability: a meta-analysis of population-based studies. Res Dev Disabil. 2011;32(2):419-36.

2. Chiurazzi P, Pirozzi F. Advances in understanding - genetic basis of intellectual disability. F1000Res. 2016;5.

3. Michelson DJ, Clark RD. Optimizing Genetic Diagnosis of Neurodevelopmental Disorders in the Clinical Setting. Clin Lab Med. 2020;40(3):231-56. 
4. Finucane BM, Myers SM, Martin CL, Ledbetter DH. Long overdue: including adults with brain disorders in precision health initiatives. Curr Opin Genet Dev. 2020;65:47-52.

5. Lee S, Rudd S, Gratten J, Visscher PM, Prins JB, Dawson PA. Gene networks associated with nonsyndromic intellectual disability. J Neurogenet. 2018;32(1):6-14.

6. Blesson A, Cohen JS. Genetic Counseling in Neurodevelopmental Disorders. Cold Spring Harb Perspect Med. 2020;10(4).

7. Bruel AL, Vitobello A, Tran Mau-Them F, Nambot S, Sorlin A, Denomme-Pichon AS, et al. Nextgeneration sequencing approaches and challenges in the diagnosis of developmental anomalies and intellectual disability. Clin Genet. 2020.

8. llyas M, Mir A, Efthymiou S, Houlden H. The genetics of intellectual disability: advancing technology and gene editing. F1000Res. 2020;9.

9. Katoh M. Molecular cloning and characterization of human WINS1 and mouse Wins2, homologous to Drosophila segment polarity gene Lines (Lin). Int J Mol Med. 2002;10(2):155-9.

10. Hatini V, Bokor P, Goto-Mandeville R, DiNardo S. Tissue- and stage-specific modulation of Wingless signaling by the segment polarity gene lines. Genes Dev. 2000;14(11):1364-76.

11. Najmabadi $H, H u H$, Garshasbi M, Zemojtel T, Abedini SS, Chen W, et al. Deep sequencing reveals 50 novel genes for recessive cognitive disorders. Nature. 2011;478(7367):57-63.

12. Akawi NA, Al-Jasmi F, Al-Shamsi AM, Ali BR, Al-Gazali L. LINS, a modulator of the WNT signaling pathway, is involved in human cognition. Orphanet J Rare Dis. 2013;8:87.

13. Sheth J, Ranjan G, Shah K, Bhavsar R, Sheth F. Novel LINS1 missense mutation in a family with nonsyndromic intellectual disability. Am J Med Genet A. 2017;173(4):1041-6.

14. Muthusamy B, Bellad A, Prasad P, Bandari AK, Bhuvanalakshmi G, Kiragasur RM, et al. A Novel LINS1 Truncating Mutation in Autosomal Recessive Nonsyndromic Intellectual Disability. Front Psychiatry. 2020;11:354.

15. Zhang X, Pan L, Shen G. [Identification of LINS1 gene variant in a patient with severe mental retardation]. Zhonghua Yi Xue Yi Chuan Xue Za Zhi. 2020;37(1):57-9.

16. McMillan HJ, Holahan AL, Richer J. Worster-Drought Syndrome Associated With LINS Mutations. Child Neurol Open. 2018;5:2329048X18791083.

17. Mazza MG, Rossetti A, Crespi G, Clerici M. Prevalence of co-occurring psychiatric disorders in adults and adolescents with intellectual disability: A systematic review and meta-analysis. J Appl Res Intellect Disabil. 2020;33(2):126-38.

18. Morgan VA, Leonard H, Bourke J, Jablensky A. Intellectual disability co-occurring with schizophrenia and other psychiatric illness: population-based study. Br J Psychiatry. 2008;193(5):364-72.

19. Benitez E, Bray SJ, Rodriguez I, Guerrero I. Lines is required for normal operation of Wingless, Hedgehog and Notch pathways during wing development. Development. 2009;136(7):1211-21.

20. Johansen KA, Green RB, Iwaki DD, Hernandez JB, Lengyel JA. The Drm-Bowl-Lin relief-of-repression hierarchy controls fore- and hindgut patterning and morphogenesis. Mech Dev. 2003;120(10):1139- 
51.

21. Bokor $P$, DiNardo $S$. The roles of hedgehog, wingless and lines in patterning the dorsal epidermis in Drosophila. Development. 1996;122(4):1083-92.

22. van Amerongen R, Nusse R. Towards an integrated view of Wnt signaling in development. Development. 2009;136(19):3205-14.

23. Katoh M, Katoh M. Molecular genetics and targeted therapy of WNT-related human diseases (Review). Int J Mol Med. 2017;40(3):587-606.

24. Ciani L, Salinas PC. WNTs in the vertebrate nervous system: from patterning to neuronal connectivity. Nat Rev Neurosci. 2005;6(5):351-62.

25. Budnik V, Salinas PC. Wnt signaling during synaptic development and plasticity. Curr Opin Neurobiol. 2011;21(1):151-9.

26. Ekici AB, Hilfinger D, Jatzwauk M, Thiel CT, Wenzel D, Lorenz I, et al. Disturbed Wnt Signalling due to a Mutation in CCDC88C Causes an Autosomal Recessive Non-Syndromic Hydrocephalus with Medial Diverticulum. Mol Syndromol. 2010;1(3):99-112.

27. Buchman JJ, Durak O, Tsai LH. ASPM regulates Wnt signaling pathway activity in the developing brain. Genes Dev. 2011;25(18):1909-14.

28. Kwan V, Unda BK, Singh KK. Wnt signaling networks in autism spectrum disorder and intellectual disability. J Neurodev Disord. 2016;8:45.

29. Krumm N, O'Roak BJ, Shendure J, Eichler EE. A de novo convergence of autism genetics and molecular neuroscience. Trends Neurosci. 2014;37(2):95-105.

30. Zhang Y, Yuan X, Wang Z, Li R. The canonical Wnt signaling pathway in autism. CNS Neurol Disord Drug Targets. 2014;13(5):765-70.

31. Gould TD, Dow ER, O'Donnell KC, Chen G, Manji HK. Targeting signal transduction pathways in the treatment of mood disorders: recent insights into the relevance of the Wnt pathway. CNS Neurol Disord Drug Targets. 2007;6(3):193-204.

32. Gould TD, Manji HK. The Wnt signaling pathway in bipolar disorder. Neuroscientist. 2002;8(5):497511.

33. Mulligan KA, Cheyette BN. Neurodevelopmental Perspectives on Wnt Signaling in Psychiatry. Mol Neuropsychiatry. 2017;2(4):219-46.

34. Peng Y, Xu Y, Cui D. Wnt signaling pathway in schizophrenia. CNS Neurol Disord Drug Targets. 2014;13(5):755-64.

35. Inestrosa NC, Montecinos-Oliva C, Fuenzalida M. Wnt signaling: role in Alzheimer disease and schizophrenia. J Neuroimmune Pharmacol. 2012;7(4):788-807.

\section{Figures}


A $\quad$ CG1216 CG1217

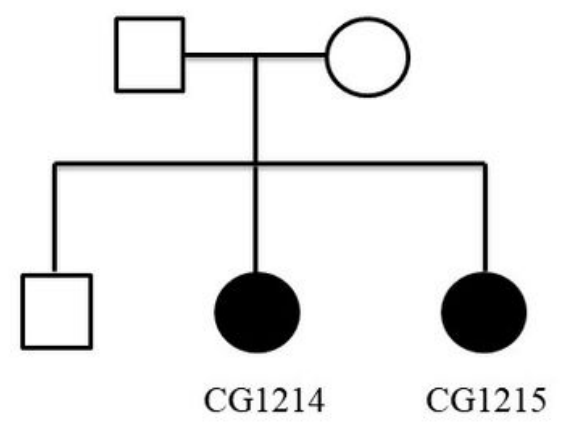

B

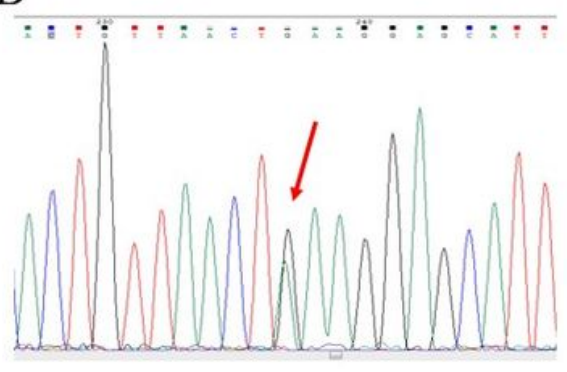

$\mathrm{C}$

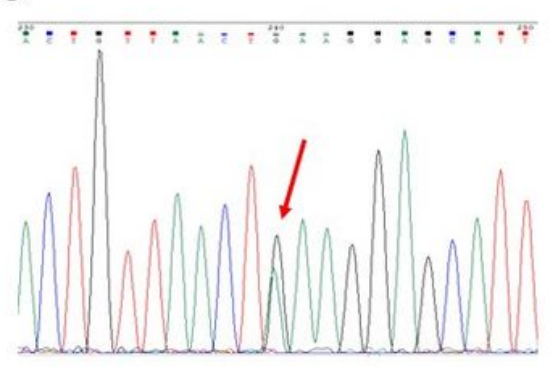

D

E
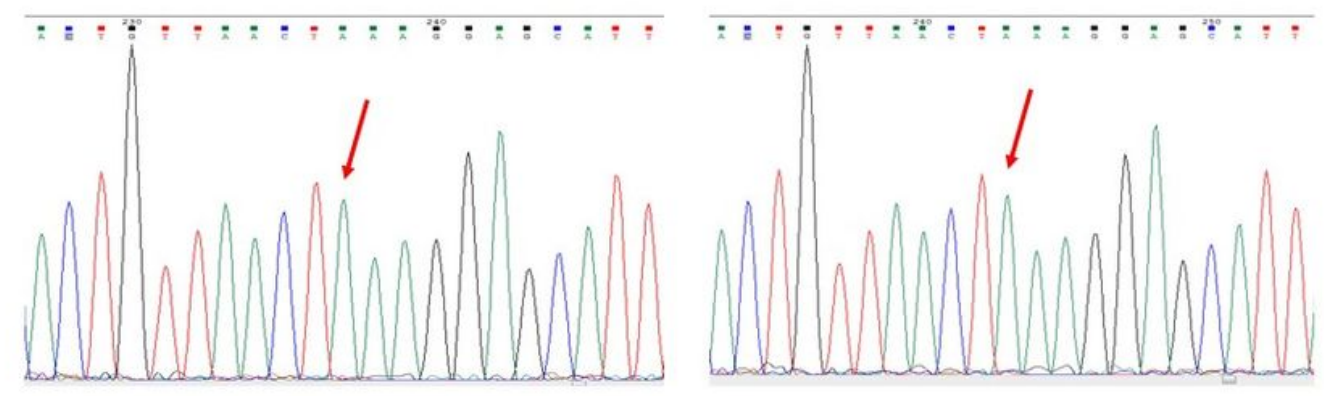

\section{Figure 1}

Pedigree and chromatogram of the GIn92X mutation of the LINS1 gene identified in this study. (A) Pedigree of the family. (B) Chromatogram of the father (CG1216) who is a heterozygous carrier of the Gto-A mutation. (C) Chromatogram of the mother (CG1216) who is also a heterozygous carrier of the G-toA mutation. (D) Chromatogram of the elder sister (CG1214) who is a homozygote of the G-to-A mutation. (E) Chromatogram of the younger sister (CG1215) who is also a homozygote of the G-to-A mutation. The red arrow indicates the position of nucleotide change. 


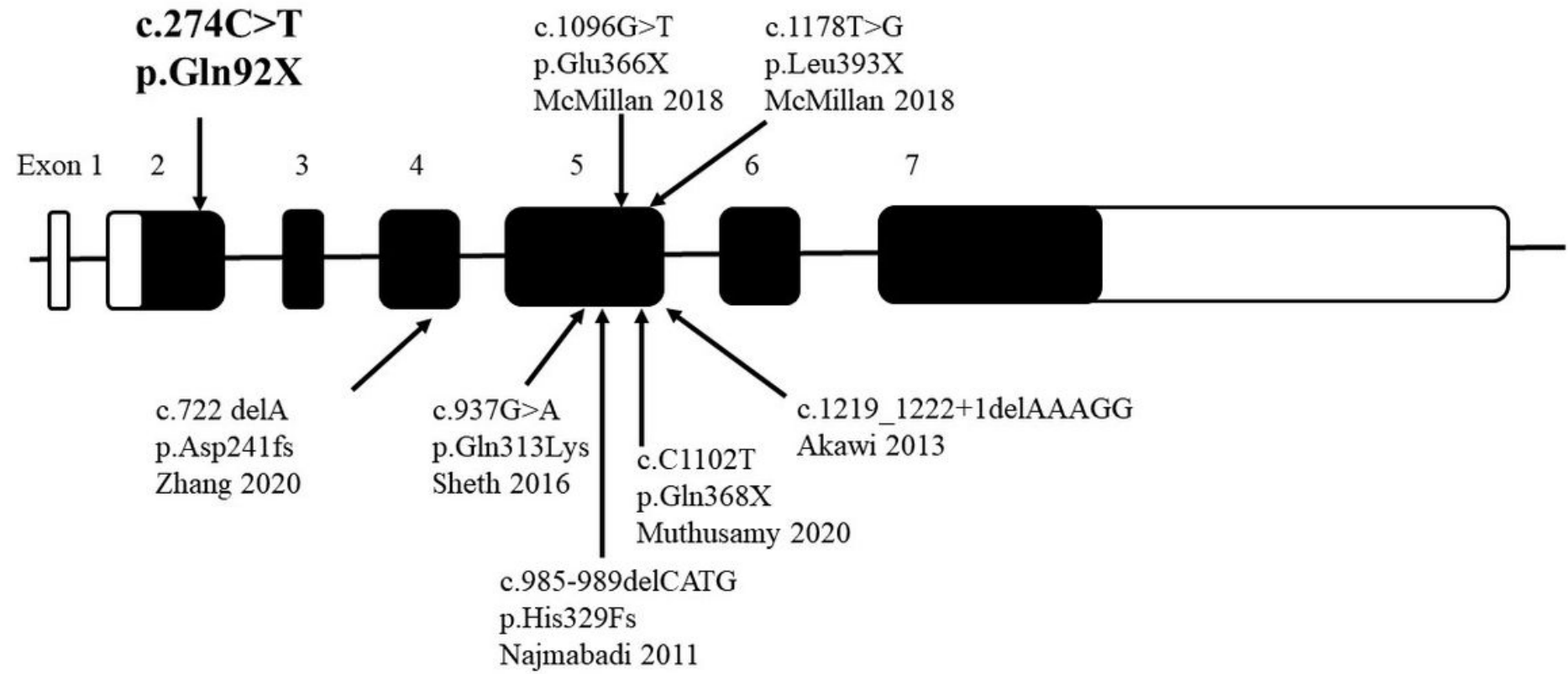

\section{Figure 2}

Schematic locations of mutations of the LINS1 gene associated with a recessive form of intellectual disability reported in the literature. The GIn92X mutation (in bold) located at exon 2 is the novel one identified in the present study. 